\title{
Inflammatory suppressive effect of prostate cancer cells with prolonged exposure to transforming growth factor $\beta$ on macrophage-differentiated cells via downregulation of prostaglandin $\mathbf{E}_{2}$
}

\author{
AKINOBU HAYASHI ${ }^{1 *}$, YOSHIFUMI S. HIROKAWA ${ }^{1 *}$, MICHIKO KAGAYA ${ }^{1}$, MASAYA FUJIWARA ${ }^{1}$, \\ MISAO YONEDA ${ }^{2}$, KAZUKI KANAYAMA ${ }^{1}$, KATSUNORI UCHIDA ${ }^{1}$, KENICHIRO ISHII $^{1}$ and TAIZO SHIRAISHI ${ }^{1}$ \\ ${ }^{1}$ Department of Oncologic Pathology, Institute of Molecular and Experimental Medicine, \\ Mie University Graduate School of Medicine, Tsu, Mie 514-8507; ${ }^{2}$ Department of Clinical Nutrition, \\ Faculty of Health Science, Suzuka University of Medical Science, Suzuka, Mie 510-0226, Japan
}

Received November 25, 2013; Accepted July 1, 2014

DOI: $10.3892 / 01.2014 .2402$

\begin{abstract}
Transforming growth factor $\beta 1$ (TGF $\beta 1$ ) regulates a variety of cellular functions, including cell growth, apoptosis and differentiation. The aim of the current study was to investigate the alterations of phenotypic events in the long-term exposure of prostate cancer (PCa) cells to TGF $\beta 1$ and its effect on macrophage-differentiated cells. The PCa cell line, PC-3, and the subclone, M1, were exposed to TGF $\beta 1$ for short- or long-term periods. TGF $\beta 1$ signaling was assessed by Smad3 phosphorylation, and non-canonical signaling was analyzed by quantitative polymerase chain reaction-based regulatory gene expression profiles. TGF $\beta 1$-exposed PCa cells were also co-cultured with phorbol 12-myristate 13-acetate (PMA)-treated THP-1 macrophages as a model of the tumor microenvironment. The phosphorylation of Smad3 in the $\mathrm{PCa}$ cells with long-term exposure was lower than that in the $\mathrm{PCa}$ cells with short-term exposure. Interleukin-6 mRNA expression in the PMA-treated THP-1 macrophages was significantly downregulated by co-culture with the PCa cells with long-term exposure. Cyclooxygenase-2 expression in the long-term
\end{abstract}

Correspondence to: Dr Yoshifumi S. Hirokawa, Department of Oncologic Pathology, Institute of Molecular and Experimental Medicine, Mie University Graduate School of Medicine, 2-174 Edobashi, Tsu, Mie 514-8507, Japan

E-mail: ultray2k@clin.medic.mie-u.ac.jp

*Contributed equally

Abbreviations: TGF $\beta 1$, transforming growth factor $\beta 1$; COX-2, cyclooxygenase-2; EMT, epithelial mesenchymal transition; $\mathrm{PGE}_{2}$, prostaglandin $\mathrm{E}_{2}$; TAM, tumor-associated macrophage

Key words: prostate cancer, transforming growth factor $\beta 1$, THP-1 macrophage, microenvironment
TGF $\beta 1$-exposed PCa cells was lower than that in the control $\mathrm{PCa}$ cells, and the production of prostaglandin $\mathrm{E}_{2}\left(\mathrm{PGE}_{2}\right)$ in the long-term TGF $\beta 1$-exposed PCa cells was also significantly lower. The results of the current study demonstrated that the long-term TGF $\beta 1$ exposure of PCa cells induces phenotypic changes, including the downregulation of $\mathrm{PGE}_{2}$ production. This indicates that prolonged TGF $\beta$-exposed PCa cells may change the cytokine production of macrophages in the tumor microenvironment.

\section{Introduction}

Cancer and the surrounding stromal cells compose the tumor microenvironment that provides opportunities for reciprocal interactions between cancer, fibroblasts, inflammatory cells and microcapillary vessels. Cytokines, including chemokines, growth factors, angiogenic factors and prostaglandins, participate in these interactions. Among these cytokines, transforming growth factor $\beta 1$ (TGF $\beta 1$ ) is a multifunctional growth factor produced by cancer cells, macrophages and fibroblasts, which exerts wide-ranging activities that affect cancer inhibition and promotion, epithelial mesenchymal transition (EMT), angiogenesis and the suppression of regulatory $\mathrm{T}$ cell function (1).

In human prostate cancer (PCa) tissues, TGF $\beta 1$ expression is increased compared with normal or benign prostate tissues, and is increased in PCa with lymph node metastasis compared with cancer without lymph node involvement (2). TGF $\beta 1$ suppresses PCa cell growth in a dose-dependent manner, but this inhibitory effect is lost at later stages $(3,4)$. Collectively, TGF $\beta 1$ is considered to be a tumor promoter in PCa tissues.

Cancer and inflammation has long been studied in close connection with carcinogenesis and cancer development. Cyclooxygenase-2 (COX-2) is the major enzyme that converts arachidonic acid into prostanoids, which are involved in a number of pathological events, including inflammation and cancer progression (5). However, the mechanistic role of COX-2 in prostate carcinogenesis remains controversial. 
One study has shown that benign prostatic disease expresses higher COX-2 than $\mathrm{PCa}(6)$, while another study reported COX-2 overexpression in $\mathrm{PCa}(7)$.

Multiple inflammatory cells and mediators are involved in cancer-related inflammation and compose elements of the tumor microenvironment (8). Tumor-associated macrophages (TAM), which are derived from monocytes, infiltrate tumor tissue, promote the invasive capacity of cancer cells and in turn, metastasis, which is correlated with a poor prognosis in patients with prostate and breast cancer (9-12). The mechanism by which TAM promotes cancer promotion is considered to involve the production of angiogenic growth factors, proteases and cytokines, including TGF $\beta$ (13). The reciprocal interactions between macrophages and the various phenotypes of $\mathrm{PCa}$ in the tumor microenvironment may be diverse. To investigate this issue, the current study examined the tumor microenvironment model of PCa, and TGF $\beta$ and THP-1 macrophages, where the PCa cells were exposed to TGF $\beta$ for a long period of time. In addition, the cytokine mRNA from THP-1 macrophages and the regulatory factors from PCa were analyzed.

\section{Materials and methods}

Cell culture and reagents. The human PCa cell line, PC-3, and the subclone, M1 (14), were cultured in RPMI 1640 (R8758; Sigma-Aldrich, St. Louis, MO, USA) with $10 \%$ fetal bovine serum (FBS; Gibco-BRL, Carlsbad, CA, USA), and TGF $\beta 1$ (555-83601; Wako Pure Chemical Industries, Ltd., Osaka, Japan) was added to the culture medium at a final concentration of $10 \mathrm{ng} / \mathrm{ml}$. The cell lines were cultured and passaged every seven days. TGF $\beta 1$ or vehicle was added to the culture medium simultaneously on each passage and kept in the medium until the next passage. The passage was repeated 10 times. TGF $\beta 1$ or vehicle long-term exposure for the PC3 and M1 cells was designated as TbL-PC3 or TbL-M1, and CoL-PC3 or CoL-M1, respectively. The cell culture of the short-term exposure was represented by overnight incubation of TGF $\beta 1$ or vehicle, and TGF $\beta 1$ was removed from the culture medium in subsequent experiments. Short-term exposure for the PC3 and M1 cells was designated as TbS-PC3 or TbS-M1, and CoS-PC3 or CoS-M1, respectively. The human acute monocytic leukemia-derived THP-1 cell line was maintained in RPMI 1640 medium supplemented with $10 \%$ FBS. The antibodies for western blot analysis and their dilutions were as follows: Rabbit polyclonal anti-human anti-Smad3 (ab28379; 1:1,000), rabbit monoclonal anti-human anti-phospho-Smad3 (S423+S425; ab52903, 1:1,000) and rabbit polyclonal anti-rat anti-COX-2 (ab15191; 1:1,000) (All Abcam, Cambridge, UK). The p3TP-Lux plasmid was kindly provided by Dr Joan Massague (15). The luciferase assay was performed as follows: The PC-3 cells were transfected with expression and reporter plasmids together with Lipofectamine (11668019; Invitrogen Life Technologies, Carlsbad, CA, USA) and harvested $24 \mathrm{~h}$ later. The firefly luciferase activity was counted using a Dual-Luciferase Reporter Assay System (E1910; Promega Corporation, Madison, WI, USA). Renilla luciferase activity was also estimated by cotransfection of the pRL-TK vector (E2241; Promega Corporation) as an internal control.

Quantitative polymerase chain reaction ( $q P C R)$. Total RNA was isolated from THP-1 macrophages using an RNA extraction kit (74104; Qiagen, Hilden, Germany). First-strand cDNA synthesis was performed using the Transcriptor High Fidelity cDNA Synthesis kit (05081963001; Roche Diagnostics $\mathrm{GmbH}$, Mannheim, Germany). qPCR was performed with the QuantiTect SYBR Green PCR kit (204145; Qiagen) according to the manufacturer's instructions. The results were analyzed by Rotor-GeneQ software (9020353; Qiagen) and normalized against GAPDH mRNA levels. The mRNA expression of 84 genes for human signal transduction molecules was analyzed by the RT ${ }^{2}$ Profiler PCR Array (PAHS-014A; Qiagen), with the cDNA synthesis and SYBR Green PCR performed as aforementioned.

Co-culture assay of the PCa cell line and THP-1 macrophages. The THP-1 cells were cultured at $5 \times 10^{5}$ cells/well in 24 -well plates and differentiated to THP-1 macrophages with $100 \mathrm{~nm}$ phorbol 12-myristate 13-acetate (PMA; P1585; Sigma-Aldrich) for two days. Following PMA removal from the culture media, the treated cells were maintained in RPMI 1640 with $10 \%$ FBS for an additional two days. The PC-3 cell line (without TGF $\beta 1$ ) was loaded in a cell culture insert (1.0- $\mu \mathrm{M}$ pore size; 353104; BD Falcon ${ }^{\mathrm{TM}}$ Cell Culture Inserts; BD Biosciences, Franklin Lakes, NJ, USA) at $1 \times 10^{4}$ cells $/ 300 \mu 1$ medium and the inserts were placed in each well of a THP-1 macrophage culture plate. After two days of co-culture, total RNA was extracted from the THP-1 macrophages and cDNA was synthesized as aforementioned.

qPCR primers. The primersequences used were as follows: interleukin (IL)-6 forward, 5'-TCAGAACGAATTGACAAACA-3' and reverse, 5'-TTGAATCCAGATTGGAAGC-3'; TNF- $\alpha$ forward, 5'-GACAAGCCTGTAGCCCATGT-3' and reverse, 5'-TCTCAGCTCCACGCCATT-3'; and IL-10 forward, 5'-GCTGGAGGACTTTAAGGGTTACCT-3' and reverse, 5'-CTTGATGTCTGGGTCTTGGTTCT-3'.

Prostaglandin $E_{2}\left(P G E_{2}\right)$ production and enzyme immunoassay. All the cells were cultured at $5 \times 10^{5}$ cells/well in triplicates of 24-well plates. The culture medium of the cells was changed to RPMI 1640 without FBS, but containing $10 \mu \mathrm{M}$ of arachidonic acid (A3555; Sigma-Aldrich). Following $2 \mathrm{~h}$ of incubation, the media were collected from each well and $\mathrm{PGE}_{2}$ production was determined by the DetectX Prostaglandin $\mathrm{E}_{2}$ Enzyme Immunoassay kit (K018-H1; Arbor Assays, Ann Arbor, MI, USA).

\section{Results}

Smad3 phosphorylation status of PCa cells in response to short-and long-term TGF $\beta 1$ stimulation. To determine whether long-term TGF $\beta 1$ exposure can modify $\mathrm{PCa}$ cell signaling events, the PCa cell line, PC-3, and subclone, M1 (14), were used. Since IL-8 expression, which is regulated by TGF $\beta$, is slightly different in PC-3 and M1 cells, we hypothesized that these cell lines may respond differently to TGF $\beta$.

A growth inhibitory effect was observed when the PC-3 cells were exposed to TGF $\beta 1$ (4); PC-3 cells express TGF $\beta 1$ target genes (16), therefore, alterations in signaling caused by TGF $\beta 1$ stimuli should be observable. 
A

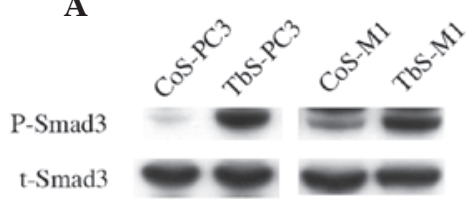

Short-term exposure

C

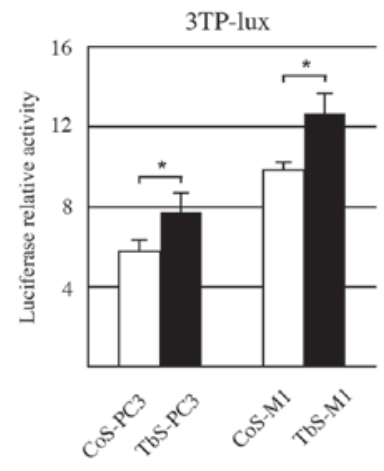

Short-term exposure
B

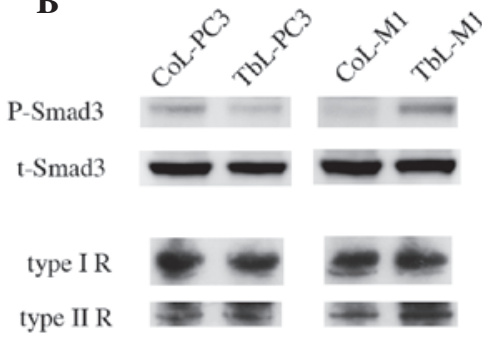

D

Long-term exposure 3TP-lux

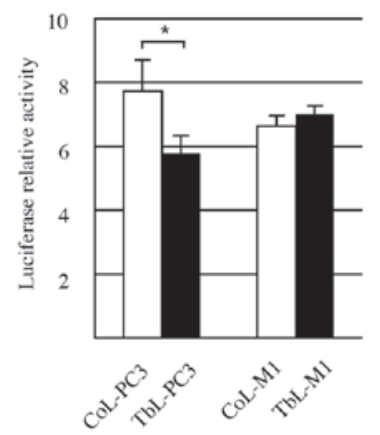

Long-term exposure

Figure 1. Smad3 phosphorylation status following short- and long-term TGF $\beta 1$ exposure and TGF $\beta 1$ target gene promoter activity. (A) Western blot analysis of P-Smad3 in short-term TGF $\beta 1$ - or vehicle-exposed PC3 and M1 cell lines. Cell line designation of TGF $\beta 1$ or vehicle short-term exposure is described in the Materials and methods section. (B, upper) Western blot analysis of P-Smad3 in long-term TGF $\beta 1$ - or vehicle-exposed cells. (B, lower) Protein expression with TGF $\beta$ types IR and IIR following TGF $\beta 1$ long-term exposure. (C and D) Smad3 target gene promoter activity was measured using the 3TP-lux luciferase reporter plasmid. Data are presented as the mean \pm standard deviation of triplicate assays and represent two independent experiments. Student's t-test was used to calculate statistical significances. * $\mathrm{P}<0.05$. TGF $\beta 1$, transforming growth factor $\beta 1$; P-Smad3, phosphorylated Smad3; t-Smad3, total Smad3; type IR, type I receptor; type IIR, type II receptor.

When the PC-3 cells were incubated with TGF $\beta 1$, Smad2 C-terminal phosphorylation (Ser465/467) was induced, but was not robust (data not shown). In the majority of the commercially available antibodies, the C-terminal phosphorylated form of Smad2 and Smad3 is not distinguishable. The anti-phospho-Smad3 antibody, described in the Materials and methods section, does not cross-react with phospho-Smad2. Therefore, TGF $\beta 1$ signaling was evaluated using the phosphorylated status of Smad3.

As predicted, robust Smad3 phosphorylation was observed in the PC-3 and M1 cell lines tested following short-term TGF $\beta 1$ exposure (Fig. 1A). To further confirm Smad3 activity, a luciferase assay was conducted using the promoter for PAI-1, a Smad3 target gene. As shown in Fig. 1C, when the PC-3 and M1 cells were exposed to TGF $\beta 1$ in the short-term, the PAI-1 promoter activity was unregulated.

Following long-term exposure to TGF $\beta 1$, Smad3 phosphorylation in the TbL-PC3 and TbL-M1 cells was highly diminished compared with that in the vehicle-exposed cells (CoL-PC3 and CoL-M1), while TGF $\beta$ receptor expression was compatible between vehicle and TGF $\beta 1$ exposure (Fig. 1B). In contrast to the short-term exposure, PAI-1 promoter activity of the PC3 cells with long-term exposure was diminished compared with the cells exposed to the control treatment (Fig. 1D). PAI-1 promoter activity of the M1 cells with long-term exposure was at a similar level to that of the cells exposed to the control treatment (Fig. 1D). These results indicated that Smad signaling is attenuated in the PC-3 and M1 PCa cell lines exposed to long-term TGF $\beta 1$ treatment.
Long-term TGF $\beta 1$ exposure of PCa cell suppresses cytokine production by THP-1 differentiated macrophages. To mimic the tumor microenvironment, the reciprocal interactions between TGF $\beta 1$-exposed PCa cells and inflammatory cells, in this case, macrophages, was examined. The activated macrophages were characterized with respect to the cytokines and receptors they produced and were designated as polarized macrophages (17). Since primary tissue macrophages are not easily obtainable, the human monocytic leukemia THP-1 cell line has been utilized in a number of studies (18-20). PMA treatment of THP-1 cells induces their differentiation into macrophage-like cells (THP-1 macrophages) that mimic the characteristics of monocyte-derived macrophages (21). As described in the Materials and methods section, cytokine production from THP-1 macrophages following reciprocal interactions with $\mathrm{PCa}$ cells was assessed by a chamber assay, where THP-1 macrophages and PCa cells were separated and could not make direct contact. When the THP-1 macrophages were co-cultured with the PC-3 cell line without any treatment, all cytokine production was increased, as previously described (22) (data not shown).

IL-6 expression from the THP-1 macrophages was significantly decreased upon co-culturing with the PC-3 and M1 cells exposed to TGF $\beta 1$ for a long-term period (Fig. 2). TNF- $\alpha$ expression from the THP-1 macrophages was also markedly suppressed in the TbL-PC3 and TbL-M1 cells compared with the control cells. IL-10 expression was not altered significantly. However, IL-6 expression was increased, rather than decreased, by the addition of TGF $\beta 1$ to the THP-1 macrophage culture (data not shown). 

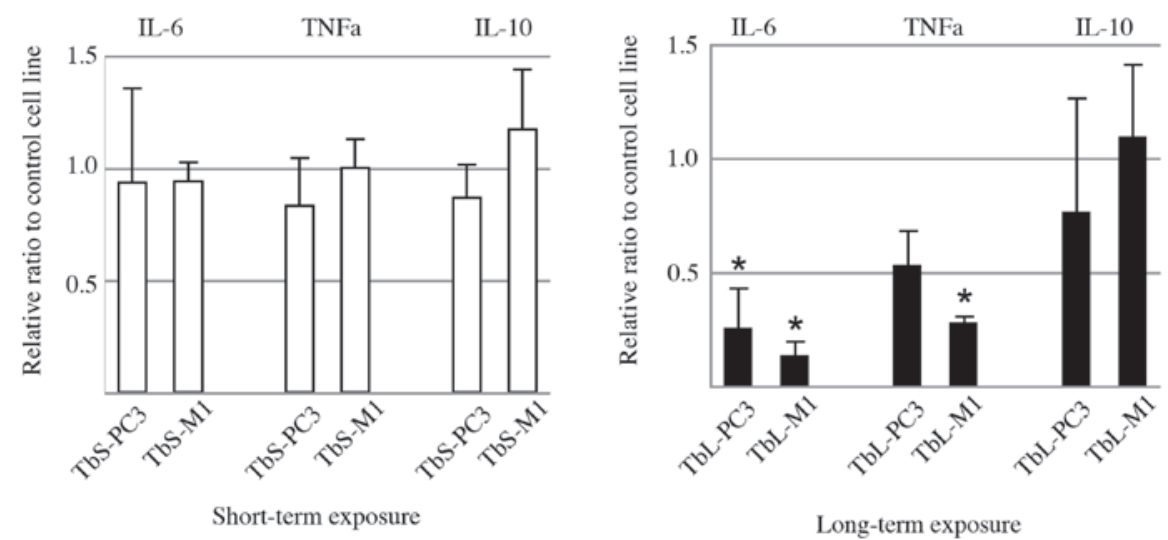

Figure 2. Cytokine production from THP-1 macrophages co-cultured with short- and long-term TGF $\beta 1$ exposure. Transcription levels of each cytokine from THP-1 macrophages with short- and long-term TGF $\beta 1$ exposure were measured by quantitative polymerase chain reaction. Relative ratios are exhibited as fold-changes compared with vehicle exposure. Data are presented as the mean \pm standard deviation of triplicate assays and represent three independent experiments. Student's t-test was used to calculate statistical significances. ${ }^{*} \mathrm{P}<0.05$ vs. vehicle exposure. TGF $\beta$, transforming growth factor $\beta$.

A
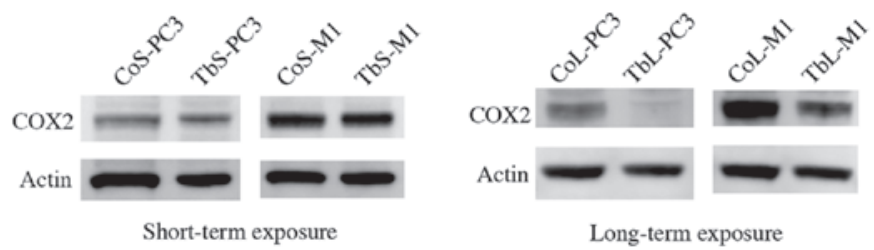

B
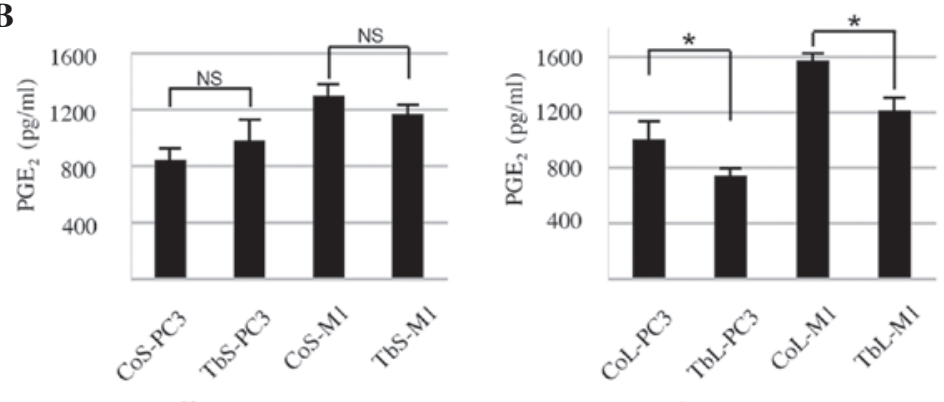

Short-term exposure

Long-term exposure

Figure 3. COX2 expression and $\mathrm{PGE}_{2}$ production by prostate cancer cells following short- and long-term exposure to transforming growth factor $\beta 1$. (A) Cell lysates from each cell line were analyzed by western blot analysis using COX-2 antibody. (B) Each cell was treated with $10 \mu \mathrm{M}$ arachidonic acid for $2 \mathrm{~h}$, and $\mathrm{PGE}_{2}$ in medium was measured by an enzyme immunoassay. $\mathrm{PGE}_{2}$ levels are expressed as equal numbers of each cell type. Data are presented as the mean \pm standard deviation of triplicate assays and represent two independent experiments. Student's t-test was used to calculate statistical significances. ${ }^{*} \mathrm{P}<0.05$ vs. vehicle exposure (CoL-PC3 and CoL-M1). COX-2, cyclooxygenase-2; $\mathrm{PGE}_{2}$, prostaglandin $\mathrm{E}_{2}$.

Since IL-6 is a key regulator in PCa progression $(23,24)$, the mechanisms of IL-6 downregulation in the THP-1 macrophages were investigated. Several growth factors, cytokines and prostanoids, such as hepatocyte growth factor (HGF), IL-1 $\beta$, IL-4 and $\mathrm{PGE}_{2}$, have been found to regulate IL-6 production from macrophages or peripheral blood monocytes (25-27). Therefore, we hypothesized that TGF $\beta 1$ suppresses or stimulates pleiotropic factor secretion from $\mathrm{PCa}$ cells and consequently downregulates IL-6 production by THP-1 macrophages. Several of these potential factors were assessed at an mRNA level by qPCR (data not shown), which showed that HGF mRNA was unchanged in the CoL-PC3, TbL-PC3, CoL-M1 and TbL-M1 cells regardless of TGF $\beta 1$ exposure (data not shown). Although HGF was reported to downregulate IL-6 production from monocytic cell lines (25), this appears to have less relevance for IL-6 reduction in THP-1 macrophages.

Next, the possibility of non-canonical signal activity was explored in the cell lines with long-term TGF $\beta 1$ exposure through use of qPCR-based array analysis, which profiled the expression of key genes that are representative of various signal transduction pathways. Overall, the PCR array analysis showed that the TGF $\beta$-related genes exhibited no marked changes in gene expression, with all changes observed being less than two-fold (Table I). Meanwhile, for the genes involved in the phospholipase $\mathrm{C}$ pathways, the expression of the COX-2 gene was downregulated in response to long-term TGF $\beta 1$ exposure. COX-2 protein expression was reduced by long-term TGF $\beta 1$ exposure in the TbL-PC3 and TbL-M1 cells (Fig. 3A). 
Table I. qPCR analysis of human signal transduction molecules

\begin{tabular}{lll}
\hline Pathway & \multicolumn{1}{c}{ Gene } & $\begin{array}{c}\text { Fold-change of } \\
\text { TbL-PC3 to CoL-PC3 }\end{array}$ \\
\hline TGF $\beta$ & CDKN1A (p21) & 1.670 \\
& CDKN1B (p27) & 0.835 \\
& CDKN2A (p16) & 1.558 \\
Phospholipase C & FOS & 0.727 \\
& ICAM1 $1 \mathrm{p} 15)$ & 0.363 \\
& NOS2A & 0.959 \\
& COX2 & 0.389 \\
\end{tabular}

${ }^{\text {aFold }}$ expression changes of TbL-PC3 to CoL-PC3 cells were calculated according to the instructions of the $\mathrm{RT}^{2}$ Profiler PCR array. COX-2, cyclooxygenase-2; TGF $\beta$, transforming growth factor $\beta$; qPCR, quantitative polymerase chain reaction.

$\mathrm{PGE}_{2}$ is known to induce IL-6 production from macrophages and is regulated by COX-2 activity (27), one of the rate-limiting enzymes for prostanoid biosynthesis (5).

The ability of the cells to produce $\mathrm{PGE}_{2}$ was also examined in the present study. The $\mathrm{PGE}_{2}$ level was not significantly different following short-term TGF $\beta 1$ exposure. However, following long-term TGF $\beta 1$ exposure, $\mathrm{PGE}_{2}$ production in the TbL-PC3 and TbL-M1 cells was reduced compared with that in the control cells (Fig. 3B).

Thus, these results indicated that $\mathrm{COX}-2$ attenuation may be responsible for the reduction in $\mathrm{PGE}_{2}$ caused by long-term TGF $\beta 1$ exposure in TbL-PC3 and TbL-M1 cells, which consequently reduces IL- 6 production in THP-1 macrophages.

\section{Discussion}

In the current study, Smad signaling was shown to be diminished in the PCa cells following long-term TGF $\beta 1$ exposure. Cytokine production from THP-1 macrophages, particularly IL-6, was downregulated upon co-culture with PCa cells, producing lower levels of COX-2 and $\mathrm{PGE}_{2}$ by long-term TGF $\beta 1$ exposure.

The dynamic function of TGF $\beta 1$ allows it to be involved in a variety of intracellular signal transduction pathways (28). Several lines of evidence support the fact that a number of TGF $\beta 1$ signal transductions are independent from Smad canonical activation (29). The underlying mechanism for the reductions in phospho-Smad3 expression following long-term TGF $\beta 1$ exposure has not yet been fully elucidated. The turnover of phospho-Smad is mediated by the specific phosphatases PPM1A, PDP and SCP1, 2 and 3, or proteasomal degradation with the ubiquitin E3 ligase, NEDD4L (30-33). Neither the PPM1A transcript nor the NEDD4L protein expression were altered following exposure of the PCa cells to TGF $\beta 1$ or vehicle (data not shown). Other unidentified phosphatases or ubiquitin ligases may therefore be involved in the suppression of phospho-Smad3.

In the tumor microenvironment, TGF $\beta 1$ is produced by a variety of cells and acts as an intercellular signaling molecule that induces the expression of cytokines and angiogenic factors, which consequently promote tumor growth, invasion and metastasis (1). Long-term reciprocal interactions between cancer cells and fibroblasts, which are a source of TGF $\beta 1$ production (34), give rise to an altered cancer cell phenotype that may affect stromal components. In the present study, the long-term exposure of the PCa cells to TGF $\beta 1$ was found to suppress THP-1 macrophage activation in a co-culture system. This result concurs with a colon cancer study in which a COX-2-degrading enzyme was upregulated by TGF $\beta 1$ (35), suggesting that the long-term exposure of PCa cells to TGF $\beta 1$ may have a similar COX-2 suppression mechanism. The current in vitro results may have relevance for physiological cancer tissues, wherein certain populations of cancer cells may control inflammatory cell function and gain survival advantages. A study revealed that when NF- $\mathrm{NB}$ signaling was repressed in TAMs, those TAMs showed cytotoxicity against tumor cells (36). In addition, normal mammary epithelial cells (MECs) exposed to TGF $\beta 1$ underwent EMT and acquired features of stromal cells. These immortalized and transformed MECs with EMT-regulated gene expression also showed increased mammosphere formation, a surrogate measure of stemness (37). Taken together, these results suggested that the long-term exposure of PCa cells to TGF $\beta 1$ may also promote a stem cell-like character. However, whether PCa with stem cell-like characteristics can suppress macrophage activity in tumors has not been fully investigated. The current in vitro results indicated the possibility of a macrophage inhibitory mechanism in the tumor microenvironment.

\section{Acknowledgements}

This study was supported by Grants-in Aid from the Ministry to Education for Science and Culture of Japan (grant no. 22591765).

\section{References}

1. Yingling JM, Blanchard KL and Sawyer JS: Development of TGF-beta signalling inhibitors for cancer therapy. Nat Rev Drug Discov 3: 1011-1022, 2004.

2. Eastham JA, Truong LD, Rogers E, et al: Transforming growth factor-beta 1: comparative immunohistochemical localization in human primary and metastatic prostate cancer. Lab Invest 73 : 628-635, 1995.

3. Kim IY, Ahn HJ, Zelner DJ, et al: Genetic change in transforming growth factor beta (TGF-beta) receptor type I gene correlates with insensitivity to TGF-beta 1 in human prostate cancer cells. Cancer Res 56: 44-48, 1996.

4. Wilding G, Zugmeier G, Knabbe C, Flanders K and Gelmann E: Differential effects of transforming growth factor beta on human prostate cancer cells in vitro. Mol Cell Endocrinol 62: 79-87, 1989.

5. Fosslien E: Molecular pathology of cyclooxygenase-2 in neoplasia. Ann Clin Lab Sci 30: 3-21, 2000.

6. Zha S, Gage WR, Sauvageot J, et al: Cyclooxygenase-2 is up-regulated in proliferative inflammatory atrophy of the prostate, but not in prostate carcinoma. Cancer Res 61: 8617-8623, 2001.

7. Gupta S, Srivastava M, Ahmad N, Bostwick DG and Mukhtar H: Over-expression of cyclooxygenase-2 in human prostate adenocarcinoma. Prostate 42: 73-78, 2000

8. Mantovani A, Allavena P, Sica A and Balkwill F: Cancer-related inflammation. Nature 454: 436-444, 2008.

9. Hagemann T, Wilson J, Kulbe H, et al: Macrophages induce invasiveness of epithelial cancer cells via NF-kappa B and JNK. J Immunol 175: 1197-1205, 2005.

10. Qian B, Deng Y, Im JH, et al: A distinct macrophage population mediates metastatic breast cancer cell extravasation, establishment and growth. PLoS One 4: e6562, 2009.

11. Lissbrant IF, Stattin P, Wikstrom P, et al: Tumor associated macrophages in human prostate cancer: relation to clinicopathological variables and survival. Int J Oncol 17: 445-451, 2000. 
12. Denardo DG, Brennan DJ, Rexhepaj E, et al: Leukocyte complexity predicts breast cancer survival and functionally regulates response to chemotherapy. Cancer Discov 1: 54-67, 2011.

13. Qian BZ and Pollard JW: Macrophage diversity enhances tumor progression and metastasis. Cell 141: 39-51, 2010.

14. Hirokawa YS, Takagi A, Uchida K, et al: High level expression of STAG1/PMEPA1 in an androgen-independent prostate cancer PC3 subclone. Cell Mol Biol Lett 12: 370-377, 2007.

15. Wrana JL, Attisano L, Cárcamo J, et al: TGF beta signals through a heteromeric protein kinase receptor complex. Cell 71: 1003-1014, 1992.

16. Park BJ, Park JI, Byun DS, Park JH and Chi SG: Mitogenic conversion of transforming growth factor-betal effect by oncogenic Ha-Ras-induced activation of the mitogen-activated protein kinase signaling pathway in human prostate cancer. Cancer Res 60: 3031-3038, 2000.

17. Mantovani A, Sozzani S, Locati M, Allavena P and Sica A: Macrophage polarization: tumor-associated macrophages as a paradigm for polarized M2 mononuclear phagocytes. Trends Immunol 23: 549-555, 2002.

18. El Fiky A, Perreault R, McGinnis GJ and Rabin RL: Attenuated expression of interferon- $\beta$ and interferon- $\lambda 1$ by human alternatively activated macrophages. Hum Immunol 74: 1524-1530, 2013.

19. Wu TH, Li YY, Wu TL, Chang JW, Chou WC, Hsieh LL, Chen JR and Yeh KY: Culture supernatants of different colon cancer cell lines induce specific phenotype switching and functional alteration of THP-1 cells. Cell Immunol 290: 107-115, 2014.

20. Danielsen PH, Møller P, Jensen KA, Sharma AK, Wallin H, Bossi R, Autrup H, Mølhave L, Ravanat JL, Briedé JJ, et al: Oxidative stress, DNA damage, and inflammation induced by ambient air and wood smoke particulate matter in human A549 and THP-1 cell lines. Chem Res Toxicol 24: 168-184, 2011.

21. Daigneault M, Preston JA, Marriott HM, Whyte MK and Dockrell DH: The identification of markers of macrophage differentiation in PMA-stimulated THP-1 cells and monocyte-derived macrophages. PLoS One 5: e8668, 2010.

22. Tsagozis P, Eriksson F and Pisa P: Zoledronic acid modulates antitumoral responses of prostate cancer-tumor associated macrophages. Cancer Immunol Immunother 57: 1451-1459, 2008.

23. Spiotto MT and Chung TD: STAT3 mediates IL-6-induced growth inhibition in the human prostate cancer cell line LNCaP. Prostate 42: 88-98, 2000.
24. Smith PC, Hobisch A, Lin DL, Culig Z and Keller ET: Interleukin- 6 and prostate cancer progression. Cytokine Growth Factor Rev 12: 33-40, 2001.

25. Kamimoto M, Mizuno S and Nakamura T: Reciprocal regulation of IL- 6 and IL-10 balance by HGF via recruitment of heme oxygenase-1 in macrophages for attenuation of liver injury in a mouse model of endotoxemia. Int J Mol Med 24: 161-170, 2009.

26. Donnelly RP, Crofford LJ, Freeman SL, et al: Tissue-specific regulation of IL-6 production by IL-4. Differential effects of IL-4 on nuclear factor-kappa B activity in monocytes and fibroblasts. J Immunol 151: 5603-5612, 1993.

27. Williams JA, Pontzer $\mathrm{CH}$ and Shacter E: Regulation of macrophage interleukin-6 (IL-6) and IL-10 expression by prostaglandin $\mathrm{E}_{2}$ : the role of $\mathrm{p} 38$ mitogen-activated protein kinase. J Interferon Cytokine Res 20: 291-298, 2000.

28. Meulmeester E and Ten Dijke P: The dynamic roles of TGF- $\beta 1$ in cancer. J Pathol 223: 205-218, 2011.

29. Moustakas A and Heldin CH: Non-Smad TGF-beta signals. J Cell Sci 118: 3573-3584, 2005.

30. Lin X, Duan X, Liang YY, et al: PPM1A functions as a Smad phosphatase to terminate TGFbeta signaling. Cell 125: 915-928, 2006.

31. Chen HB, Shen J, Ip YT and Xu L: Identification of phosphatases for Smad in the BMP/DPP pathway. Genes Dev 20: 648-653, 2006.

32. Sapkota G, Knockaert M, Alarcón C, et al: Dephosphorylation of the linker regions of Smad1 and Smad2/3 by small C-terminal domain phosphatases has distinct outcomes for bone morphogenetic protein and transforming growth factor-beta pathways. J Biol Chem 281: 40412-40419, 2006.

33. Gao S, Alarcón C, Sapkota G, et al: Ubiquitin ligase Nedd4L targets activated Smad2/3 to limit TGF-beta signaling. Mol Cell 36: 457-468, 2009.

34. Bissell MJ and Radisky D: Putting tumours in context. Nat Rev Cancer 1: 46-54, 2001.

35. Yan M,Rerko RM,Platzer P, et al: 15-Hydroxyprostaglandin dehydrogenase, a COX-2 oncogene antagonist, is a TGF-beta-induced suppressor of human gastrointestinal cancers. Proc Natl Acad Sci USA 101: 17468-17473, 2004.

36. Hagemann T, Lawrence T, McNeish I, et al: 'Re-educating' tumor-associated macrophages by targeting NF-kappaB. J Exp Med 205: 1261-1268, 2008.

37. Mani SA, Guo W, Liao MJ, et al: The epithelial-mesenchymal transition generates cells with properties of stem cells. Cell 133: 704-715, 2008. 